\title{
A CASE OF PRIMARY INFECTION OF THE PUERPERAL UTERUS BY DIPLOCOCCUS PNEUMONIA.*
}

By ALEXANDER G. R. FOULERTON, F.R.C.S., Lecturer on Bacteriology to the Middlesex Hospital, and W. F. FICTOR BONNEY, M.S., M.D. (Lond.), F.R.C.S., Assistant Physician to the Chelsea Hospital for Women.

In the course of an investigation into the bacteriolngy of cases of puerperal fever we have up to the present time met with only one in which an infection by the pneumococcus (Diplococcus pneumonia) has occurred; and the literature relating to puerperal fever shows that such infection is of sufficiently rare occurrence to justify us in recording this case in the Transactions of the Society.

A woman, aged 24 years, was seen in consultation on December 10 th, and the following history was given :--

The patient had been delivered of her first child ten days previously, after a rather difficult labour in which the use of forceps was necessitated by a contracted pelvis. On the third day after delivery there was a sharp rise of temperature, which during the next seven days ranged between $103^{\circ} \mathrm{F}$. and $105^{\circ} \mathrm{F}$. Shortly after the onset of the fever it was noticed that the abdomen was tender towards its lower part. The patient vomited occasionally and rigors occurred. Vaginal douches and warm fomentations to the abdomen werc ordered, but the circumstances of the patient were such that the treatment prescribed could not be carried out efficiently.

When seen on the tenth day after delivery, the seventh day of the fever, the patient's condition was as follows:-

Her face was haggard and of sallow complexion; her mind was quite clear, and she spoke with a strong voice. The temperature was $105^{\circ} \mathrm{F}$.; the pulse rate was over 150 ; the respiratory rate was not markedly increased. The lower part of the abdomen was tender on palpation, and an indefinite fulness could be distinguished there, more marked and extending higher up on the right side. The lochial discharge was of a brown colour and offensive odour. A perineal laceration had been united by suture immediately after delivery, but the union had broken down and the perineum now presented a wound with swollen and everted edges, and a sloughing surface. There was a large sloughing laceration of the anterior lip of the cervix, extending into the anterior vaginal fornix. The outline

* Read at a Meeting of the Obstetrical Society of London, April lst, 1903. 
of the uterus was obscured by a considerable pelvic effusion in which the organ was embedded and fixed. The effusion was of larger extent on the right side. An examination of the heart and lungs showed the absence of any signs of local disease.

Under the circumstances it was decided to transfer the case to a hospital, and the patient was admitted into the Temperance Hospital where she came under the care of Dr. Addinsell; and we have to express our thanks to Dr. Addinsell for his kindness in supplying us with further details as to the history of the case and as to the findings at the examination made after death. The patient sank rapidly after admission into the Hospital, and died on December 14th, the fifteenth day after delivery.

Autopsy. From Dr. Addinsell's notes we gather the following particulars:-

On opening the abdomen it was seen that there was some distension of the intestines and a general injection of the peritoneal membrane. There was evidence of localised peritonitis, by which the contents of the pelvis and some coils of intestine in the right iliac region had been bound together by adhesions of recent formation. There were collections of pus and turbid serous fluid between the adherent coils of intestine. The right broad ligament especially was thickened by effusion into its substance and extended as a swollen mass into the right iliac fossa. The uterus was in a condition of subinvolution, its walls were thickened and softened, and in the uterine cavity there was a small piece of placental tissue still adherent.

There was a lacerated wound of the cervix. The kidneys were of normal appearance, the spleen was slightly enlarged and firm, the liver was pale and somewhat soft. The heart was of normal appearance; there was some blood-stained fluid in the right pleural sac, and some adhesions had formed in the left sac. The base of each lung was congested, but there was no sign of pneumonic consolidation.

Bacteriological examination. When the patient was seen on Dec. 10th, material for bacteriological examination was obtained from as high up the cervical canal as it was possible to pass a swab. We were not able in this case as in most of the other cases of puerperal fever which we have investigated, to pass the swab completely into the uterine cavity.

The microscopic examination of cover-glass preparations from the swab, stained by Gram's method, showed the following organisms :- -

1. A number of diplococci with distinct capsules, which stained by Gram's method and morphologically resembled Diplococcus. pneumonia. 
2. Groups of Staphylococci which stained by Gram's method.

3. A bacillus which did not stain by Gram's method, and which morphologically resembled the Bacillus coli communis subsequently isolated.

The material on the swab was then rubbed up in peptone broth and the resulting emulsion was plated out. From the agar plates thus prepared three organisms corresponding with those recognised in the stained preparations were isolated.

1. Diplococcus pneumonia.

2. A staphylococcus corresponding in general character with S. pyogenes albus.

3. Bacillus coli communis. A small quantity of the emulsion made from the swab was injected into the peritoneal sac of a mouse. The animal died in 18 hours after inoculation, and a pure culture of Diplococcus pneumonice was obtained from its peritoneal juice and from the heart blood. Three other mice received intra-peritoneal injections of 48 hours old cultures in broth of the organism isolated from the agar plates. In each case a dose of 25 c.c. of the broth culture was injected. Of these :---

Mouse ii. was obviously ill 24 hours after inoculation but recovered. The animal was killed on the fifth day, and culture tubes inoculated from the peritoneal juice and from the heart blood remained sterile.

Youse iii. died 18 hours after inoculation. A pure culture of Diplococcus pneumonia was obtained from the heart blood and peritoneal juice.

Mouse iv. This animal died five days after inoculation. In this case also pure cultures of Diplococcus pneumonia were obtained from the peritoneal juice and from the heart blood.

The bacteriological record of the case is incomplete, inasmuch as we had no opportunity of examining the peritoneal effusion found after death; but we think that there can be no reasonable doubt but that the case was primarily one of pneumococcic infection; the other organisms which we isolated, $S$. pyogenes albus and $B$. Coli communis, represented casual secondary infection. We regard the infection as a primary one of the genital passages, since neither during life nor after death was there any evidence of any preceding lung infection by Diplococcus pneumonia.

In contrast with this case of primary infection of the genital passage by Diplococcus pneumonia we may mention another in which a bacteriological examination was recently made by one of us, and in which the presence of this organism in the uterus was clearly the result of a general infection following pneumonia. 
The patient, a woman aged 31 years was admitted into the Middlesex Hospital with definite clinical symptoms of lobar pneumonia on October 6 th, 1902 . She was about $7 \frac{1}{2}$ months pregnant, and on the second day of her illness premature labour occurred. She died on the eighth day of her illness, and at the examination made after death a lobar pneumonia of the right upper lobe was found. The uterus contained some brownish fluid which, when stained, showed a large number of organisms morphologically resembling Diplococcus pneumoniæ. Pure cultures of Diplococcus: pneumonice were obtained from the lung affected, from the heart blood, from the spleen, from the fluid in the uterus, and from elsewhere.

As we said at the commencement a primary infection of the puerperal uterus by Diplococcus pneumonice appears to be an event of rare occurrence. Doyen appears to be the only author who has recorded the presence of Diplococcus pneumonia in the healthy vagina, and he only found it on one occasion; and this although the organism is a frequent parasite of other healthy mucous membranes.

Weichselbaum ${ }^{1}$ and Bar and Tissier ${ }^{2}$ quote cases of primary infection of the genital passages during the puerperium, but the latter writers do not bring forward adequate proof of the nature of the infection in their cases, as the bacteriological diagnosis was formed merely on the examination of cover glass specimens prepared from the lochial discharges.

Another condition in which a pneumococcic infection of the uterus may occur is that recorded by Colin. ${ }^{3}$ In this case severe metrorrhagia came on some three months after an abortion. On the eighth day after the commencement of the hæmorrhage the patient came into hospital and the uterus was found to be enlarged and tender. Symptoms of meningitis followed and the patient died.

At the autopsy there were found suppurative endometritis with retention of placental tissue, together with endocarditis, suppurative meningitis and softening infarcts in the spleen. From all the lesions cultures of Diplococcus pneumonia were obtained.

Finally several writers claim to have identified Diplococcus pneumonice in cases of salpingitis either following childbirth or when secondary to a vaginitis.

Thus Witte claims to have identified the organisms in four out of 39 cases of salpingitis examined. Hartmann and Morax detected them in two out of 33 cases examined, and Zweifel, Trommel and Wertheim, have each recorded a single case of the sort.

1. Wien. Klin. Wochenschrift, No. 28, 1888.

2. Sérotherapie dans l'infection puerpérale, L'Obstétrique, pp. 97-204, 1896.

3. Mïnchener Medicinische Wochenschrift, p. 1338, 1899. 